Running head: DYNAMICS OF AROUSAL AND ATTENTION

Infant attention is dynamically modulated with changing arousal levels

de Barbaro, K.(1), Clackson, K.(2) \& Wass, S.(3)*

Medical Research Council Cognition and Brain Sciences Unit, Cambridge, UK

1 - Georgia Institute of Technology

2 - University of East London

3 - University of Cambridge

* - Corresponding author

Correspondence address: University of East London, Water Lane, London, E15 4LZ

Email: s.v.wass@uel.ac.uk

Acknowledgements: This work was supported by Medical Research Council intra-mural funding for K.B. and a British Academy Postdoctoral Fellowship to S.W. Thanks to Peter Watson for advice on statistics. 


\title{
Infant attention is dynamically modulated with changing arousal levels
}

\begin{abstract}
Predominant accounts explaining links between early looking behavior and later cognitive outcomes emphasize static individual differences in information encoding; however, work from Aston-Jones and colleagues suggests that looking behavior may be dynamically influenced by autonomic arousal. To test the Aston-Jones model, a 20-minute testing battery constituting mixed photos and cartoon clips was shown to 53 typical 12-month-olds. Look duration was recorded to index attention, and continuous changes in arousal were tracked by measuring heart rate, electro-dermal activity and movement levels. Across three analyses we found that continuous changes in arousal tracked simultaneous changes in attention measures, as predicted by the Aston Jones model. We also found that changes in arousal tended to precede (occur before) subsequent changes in attention. Implications of these findings are discussed.
\end{abstract}

Keywords: look duration, arousal, dynamic, naturalistic, attention, individual differences 


\section{Introduction}

Measures of looking duration, administered to human infants, show relative stability across testing sessions (Colombo \& Mitchell, 1990). Moreover, measures of look duration collected in the first year show low- to mid-level predictions for developing IQ and executive functions (Colombo \& Mitchell, 2009). Current theoretical accounts explaining this link highlight the static and stable nature of attentional activity. The dominant account is based in information processing theories developed in the 1960s drawing on the metaphor that the mind is a computer (Colombo \& Mitchell, 2009; Sokolov, 1963). At the heart of information processing theories is the notion that individual stimuli each "contain" discrete number of "bits" of information. This allows theorists to claim that infants who look away from a stimulus faster have encoded all of its "bits" faster than infants who look for longer periods of time at the same stimulus (Rose, Feldman, \& Jankowski, 2002).

Neurobiological models of attention, derived from animal studies, take a different approach. They emphasize that dynamic, ongoing changes in an individual's state of anticipatory readiness can lead to dynamic changes how individuals distribute their attention to their environments (Tang, Rothbart, \& Posner, 2012). Sympathetic nervous system activity has neuromodulatory effects on frontal areas critical for attention and executive functions (Arnsten, 2009; Arnsten, 1998; Arnsten $\& \mathrm{Li}, 2005)$. In particular, changes in the firing rates of norepinephrine neurons in the Locus Coeruleus, a brainstem arousal nucleus, have been shown to modulate a continuum of attentional vigilance (Aston-Jones, Chiang, \& Alexinsky, 1991; Aston-Jones, Rajkowski, \& Cohen, 1999). The Aston-Jones model of attention AJMA) states that at very low levels of arousal animals are relatively unresponsive to changes in external stimuli, whereas at high levels animals are highly sensitive to peripheral changes, showing a high rate of rapid reorientations to novel stimuli and 
scanning behaviors. Moderate levels of arousal balance the sensitivity to peripheral stimuli, allowing animals to focus on a target stimulus even in the presence of some peripheral distractors (Aston-Jones \& Cohen, 2005; Rajkowski, Kubiak, \& Aston-Jones, 1994). Theoretically, dynamic changes in arousal are thought to track the relative uncertainty of the environment. In more uncertain environments, arousal is upregulated, leading to increased sensitivity to the surroundings and in turn to reductions in uncertainty (Aston-Jones \& Cohen, 2005; Yu \& Dayan, 2003).

Dynamic relations between arousal and attention are also apparent in the developmental literature. For example, within the information processing (IP) tradition, Sokolov posited that novel stimuli induce an orienting response (Sokolov, 1963). As part of this response, acute decelerations in measures of arousal such as heart rate, mediated by increases in parasympathetic activity, allow infants to focus on novel stimuli. As infants become familiarized with, or learn, stimulus properties, the orienting response decreases, concomitant with infants' decreasing attentiveness to the stimulus. While the IP account is not mutually exclusive with the AJMA, they differ in their focus. For example, IP accounts consider physiological change as an index of cognitive ability: arousal changes are a function of the match/mismatch between internal and external representations of a stimulus (e.g. Colombo \& Mitchell, 2009; Richards \& Casey, 1991). By contrast, the AJMA provides a more agnostic framework as to the types of factors that may increase arousal (Aston-Jones \& Bloom, 1981), ranging from internal to external as well as stateor trait-based factors. In particular, infants' arousal may change due to factors unrelated to stimulus processing as traditionally conceptualized, and may also thereby affect attentional processing. Additionally, the AJMA highlights the effects of increases as well as decreases in arousal levels 
on subsequent attentional activity, thus highlighting the bidirectional relations between arousal and attention.

Such links have been considered within the developmental attention-regulation literature, where too much stimulation is thought to lead to increases in arousal, in turn leading to distraction or difficulties focusing attention (Carlson, Jacobvitz, \& Sroufe, 1995; Karmel, Gardner, \& Magnano, 1991). Karmel, Gardner and colleagues have examined changes in attention and learning before and after activities known to affect arousal levels, such as breastfeeding. After breastfeeding infants showed preference for more stimulating, higher frequency stimuli (Gardner \& Karmel, 1995) as well as increased novelty preference following repeated exposure to a stimulus (Geva, Gardner, \& Karmel, 1999), as would be predicted based on their decreased arousal. Redirecting attention can also affect arousal levels, such as when an infant averts their gaze away from a stranger, leading to a temporary decrease in heart rate (Field, 1981; Waters, Matas, \& Sroufe, 1975). For these reasons, the regulation of arousal has been described as critical in developing attentional circuits, and vice versa (Karmel et al., 1991). Drawing from and supporting these theories, both over- and under- arousal, as well as levels of reactivity, have been linked with clinical attention disorders such as ADHD (Freibergs \& Douglas, 1969; Hastings \& Barkley, 1978; Zentall, 1975). Additionally, chronic exposure to stress has been shown to have lasting impacts on cognitive function (Blair et al., 2011), but the impacts of acute changes in arousal on basic cognitive processes are not well understood. Thus, investigating the relations between arousal and attentional behaviors can provide insight into the mechanisms that may bridge early individual differences in stress-system activity to cognitive development. 
Of note, studies have indicated that arousal-attention relations may be limited to infants of four months and younger (Gardner \& Karmel, 1995), suggesting that the link between arousal and attention may only be present early in development. The lack of effect in older infants is thought to reflect the maturation of cortical attentional systems and a resultant shift to more top-down modulation of attention (Bronson, 1974; Johnson, 1990). However, it may also be the case that the specific paradigms used may no longer be valid for manipulating arousal or assessing related changes to attention at older ages. Additionally, the infants' developing regulation strategies may allow them to more quickly recover from high- and low- arousal states, making the relations with learning and attention measures collected even just minutes later much more noisy. Thus, the current literature may not adequately test the dynamic relations between arousal and attention in older infants.

We previously tested some of the behavioral predictions of the AJMA in a rich naturalistic environment with many potential distractors (de Barbaro, Chiba, \& Deák, 2011). We assessed 67 month old infants' responsivity to peripherally located videos via a number of features previously used to classify infants as "fast" or "slow" lookers, including duration of fixations, reorientation speed, and time spent looking at video stimuli. Infants who showed "faster" responsivity to peripheral videos showed less habituation to these monitors over trials relative to their slowerlooking peers. Traditional information processing models suggest that fast-looking infants learn faster and should therefore habituate more rapidly to trial structure, which was inconsistent with our data. However, the AJMA could account for these dynamic changes in looking to monitors over trials. In particular, AJMA suggests that fast-looking infants may be in a highly vigilant stimulus-driven attentional profile and may not habituate to repeated presentations of stimuli while 
uncertainty or arousal remains high. In keeping with this, we found that those infants classified as "slow" lookers, or less vigilant, spent more time overall looking to less salient stimuli such as floors or their hands. These results could not be accounted for by traditional models of infant attention. However, this study did not include any measures of arousal. Thus it remains to be shown that dynamic fluctuations in arousal within individuals are associated with simultaneous changes in attention.

The current study considers the relations between dynamic measures of arousal and attention in 12-month-old infants. Based on the Aston-Jones model, we hypothesized that, individual by individual, periods of elevated arousal would be associated with shorter durations of looks, indexing a faster or more vigilant attentional profile, whereas periods of reduced arousal would be associated with longer looks, indexing a slower attentional profile.

We examined how dynamic changes in arousal relate to dynamic changes in attention in three ways. Across all three analyses, we utilized a composite measure of continuous peripheral arousal activity, including head and foot velocity, heart rate and electrodermal activity. We have considered the dynamic relations between these different measures of arousal in two recent reports (XXXX; XXXX). In general, these individual measures of arousal were found to be highly correlated across multiple temporal scales, including second-by-second changes as well as at baseline periods and in response to attentional stimuli. For the present paper we have pooled them, therefore, into a single composite measure. 
In our first analysis we use time-series analyses to look at correlations in changing arousal and attention measures across an entire 20-minute testing session, composed of a battery of mixed static and dynamic infant-appropriate animations and TV clips. Attention was indexed by measuring look duration, the duration of looks to, vs away from, the screen. This analysis also allowed us to examine whether changes in arousal tended to occur before or after changes in attention. Neuroanatomical work indicates that changes in arousal can drive changes in looking behaviors, thus this analysis allows us to examine evidence for these temporal relations in addition to the simple correlations. We hypothesized that, if the AJMA is correct, we would observe that periods of higher arousal within a session would be associated with shorter looks, and that periods of lower arousal within a session would be associated with longer looks.

Analysis 2 investigates potential effects of stimulus dynamics on arousal-attention relations. Our entire session, which was used as the basis for Analysis 1, was comprised of different types of tasks, some with static stimuli and others with dynamic stimuli. One possibility is that any covariation observed between arousal and looking activity might be independently associated with differences in the task stimuli. In particular, past work indicates that infants show longer looks while observing complex or dynamic images, and shorter looks while observing static images (Courage, Reynolds, \& Richards, 2006; XXXXXX). Thus, to control for the potential effects of stimuli on looking and arousal, in Analysis 2 we also assessed whether similar relations could be observed within a single task in which static images were repeatedly presented to infants. This way we can ensure that effects are not driven by differences in task- and stimulus-type across the sessions. We hypothesized that, if the AJMA is correct, consistently 
negative correlations would be observed between the level of arousal during a look, and the duration of that look.

For our final analysis we examined the optimal timescale for characterizing arousal-attention dynamics. Traditionally, arousal research has distinguished between tonic and phasic shifts in arousal. Tonic activation refers to low frequency shifts in arousal, or slow shifts in the overall baseline of activity, whereas phasic activity refers to faster or higher frequency fluctuations in arousal. However, no clear cut-off exists between tonic and phasic shifts. In practice, a baseline is typically calculated at the start of recording, and a single reactivity score is calculated based on the difference between this and a period of challenge. However, slow-moving trends in arousal activity that occur during the testing session may also systematically influence changes in attention (Aston-Jones \& Cohen, 2005). Our previous analyses examined changes at only relatively short timescales. Thus, in order to examine the optimal timescale for characterizing arousal-attention dynamics, we compared a set of models across a range timescales of activity, from short epochs that could capture rapid changes, to longer epochs (up to six minutes in duration) that could capture much slower shifts in arousal. To examine the relative predictive values across these varied timescales of activity we again considered the continuous arousal and look duration data recorded over the 20-minute testing session, as presented in Analysis 1 . We hypothesized that, if the AJMA were correct, then short-term (phasic) changes in arousal would explain more of the variance in look duration than baseline arousal levels alone.

\section{Methods}

\section{Participants and study design}


All participating infants $(\mathrm{N}=52)$ completed a mixed testing battery to assess how look duration and arousal change across a 20-minute testing session. All infants were typically developing. The battery consisted of a mixture of photographs (such as pictures of another child's face, or handdrawn images) and video clips (child-appropriate home movies and excerpts from childrens' television programs). The maximum length of each individual stimulus was 45 seconds, and most were less than 20 seconds. Exactly the same stimuli were presented to all infants.

Infants were seated on their caregivers' laps during recording. No explicit instructions were given to the caregiver, but they were requested not to talk during testing. Viewing materials were presented using a Tobii TX300 eyetracker subtending approximately $30^{\circ}$ of visual angle. Stimulus presentation was performed using Matlab, Psychtoolbox and the Matlab Tobii SDK. Stimuli were presented continuously. Breaks were permitted during testing if the child became agitated or the parent requested it. This occurred rarely $(<10 \%$ of cases $)$. In these cases, recording and stimulus presentation were discontinued and only recommenced when the infant was in an alert and interested state. The average age of the participating infants was 12 months (mean age in days: 357, SD: 39, range: 315-501), and the sample comprised 27 males and 25 females.

\section{Data reduction}

Behavioral measures - session look duration. Analyses 1 and 3 were based on look duration data recorded across the entire testing battery. Look durations index how long the child looked at the screen before looking away from the screen. See Supplementary Materials for further details of how this was calculated. 
Habituation task look duration - Analysis 2 focused on data from three blocks of one particular task within the battery, a habituation task (e.g. Colombo \& Mitchell, 2009). In this task a static image a picture of a child's face) was presented repeatedly to infants until two consecutive looks had taken place that were less than $50 \%$ of their longest look to the image (following Colombo \& Cheatham, 2006). Arousal indices were measured concurrently with looking activity. Look durations were defined in the same way as for Analyses 1 and 3 above. See Supplementary Materials for further details.

Arousal composite. Electro-cardiogram (ECG), electro-dermal activity (EDA), peripheral accelerometry and head velocity data were combined to index arousal. In our recent previous research we have examined interactions between these four measures and found strong patterns of covariation between them (XXXX; XXXX). Therefore these four measures were used as the basis for the arousal composite - by first calculating z-scores for each measure, and then averaging the z-scores. Further details on the recording, and data reduction, are given in the Supplementary Materials.

\section{$\underline{\text { Results }}$}

$\underline{\text { Analysis } 1 \text { - Arousal- attention fluctuations across whole session }}$

If the AJMA is correct, we predicted that we would observe that phasic (short-term) increases in arousal would be associated with phasic (short-term) decreases in look duration, and vice versa. To investigate the dynamic relations between arousal and attention across the entire session we used common timeseries analysis techniques. In particular, we examine the average correlation between windows of arousal and attention values as they vary throughout the session, known as a 
cross-correlational analysis. Additionally, we examine how changes in arousal may predict later changes in attention, or, in other words, whether changes in arousal tend to occur before or after changes in attention, and vice versa. This is known as a lagged cross-correlational analysis. Finally, we utilize the same techniques to assess how well each variable predicts itself over time, reflecting the stability of each measure. This technique, known as an autocorrelation, is necessary for interpreting the significance of cross-correlational results.

To calculate cross-correlations, looking and arousal data were time-synchronized, and binned into one-second epochs. For simple cross-correlations the correlation was calculated across all pairs of time-locked i.e. simultaneously occurring) arousal and look epochs, using a Spearman's rank order correlation. This allowed us to examine whether epochs of high arousal are associated with shorter look durations. The value obtained is plotted as time " 0 " $(\mathrm{t}=0)$ in the cross-correlation. To examine lagged cross-correlations, we examine the correlations between non-simultaneous pairs, that is, considering the correlation between epochs at increasing lags from one another. To obtain the value plotted at a single lag (or lag-time $\mathrm{t}=-1$ ), for example, the epochs created from the arousal data were shuffled one second backwards relative to the look duration epochs, and all lagged pairs of data were entered into a correlation. In this way, we can estimate how the association between two variables changes when we increase the time-lag between them. The procedure for calculating an auto-correlation is identical to that for a cross-correlation - except that, instead of comparing one variable with another, the relation of that variable with itself was measured. Using a standard procedure, (Clifford, Richardson, \& Hemon, 1989) the significance levels for cross-correlations are adjusted based on the outputs of the autocorrelation which can be used to approximate the effective 
sample size (i.e. number of statistically independent values) for each series. See Supplementary Materials for further details of how these were calculated.

Figures 1a) and b) show the results of the auto-correlation analyses. The y-axes show the correlation observed between that variable and itself thus, perfectly correlated at time-lag $=0$, and the $\mathrm{x}$-axis the time-lag. A sharper fall-off indicates less predictive value at increasing lags, or a faster-changing measure. Statistically significant auto-correlations were observed for arousal Figure 1a) at all time lags up to 83 seconds. This indicates that on average, arousal levels in any one second epoch are significantly associated with arousal levels up to 83 seconds before and after that moment. For look duration Figure 1b), significant relations were observed at time intervals less than 37 seconds.

Figure 1c) shows the results of the cross-correlation analysis. The $\mathrm{Y}$-axes show the strength of the relation between the two variables at the various time lags, reported on the $\mathrm{X}$-axes. The negative value at the lag $\mathrm{t}=0$ indicates that second by- second fluctuations in arousal were negatively correlated with simultaneous fluctuations in look duration $(\mathrm{r}=-.22)$ as hypothesized by the Aston Jones model. The correlations decrease as the lags increase in both directions. However, it can be seen that the significance of these correlations is markedly asymmetric around the lag $\mathrm{t}=0$ point, indicating that changes in arousal tend to occur before changes in look duration. Specifically, arousal values occurring 2 through 25 seconds prior to any given epoch of looking duration data were found to significantly $(\mathrm{p}<.05)$ predict that epoch of look duration. In contrast, the values of arousal occurring after any given epoch of looking duration data were not significantly associated. Thus, changes in look durations do not predict upcoming shifts in arousal at any time-lag. 
INSERT FIGURE 1 HERE

\section{Analysis 2 Arousal- attention fluctuations within the habituation task}

The previous analysis used look duration data obtained during the administration of a testing battery consisting of a mixture of static and dynamic stimuli. To control for the potential effects of stimuli on looking and arousal, we also assessed whether similar relations could be observed in a single task in which the same static images were repeatedly presented to infants. We hypothesized that, if the AJMA is correct, consistently negative correlations would be observed between the level of arousal during a look, and the duration of that look.

Infants' individual looks towards the stimuli were recorded during a habituation experiment, together with their arousal level relative to baseline) during that look. In total, an average of 15.6 $(\mathrm{STD}=6.4)$ looks were recorded per infant, each lasting an average of $8.0(\mathrm{STD}=2.6)$ seconds. For each infant, we examined how individual looks recorded related to the arousal levels recorded during those individual looks. Since not all data were found to be parametrically distributed, a Spearman's rank order correlation was used. Based on the Aston-Jones literature we hypothesized that negative correlations would be consistently observed across the infants.

Figure 2 shows the results of this analysis. It can be seen that, in almost all participants, negative regression coefficients were observed. This indicates that infant-by-infant, individual look duration can be predicted by the arousal level within that particular look. Within a single task, looks in which infants had higher arousal levels were associated with shorter look durations. A t- 
test was conducted to assess whether the results observed differed significantly from zero. A highly significant effect was observed $\mathrm{t}(50)=6.8, \mathrm{p}<.001,95 \%$ CI ([-.33 to -.18]). This indicates that consistently negative relations are observed between look durations and the average level of arousal during that look.

INSERT FIGURE 2 HERE.

\section{Analysis 3-Optimal timescales for capturing arousal-attention dynamics}

The AJMA predicts that short-term (phasic) changes in arousal should explain more of the variance in look duration than baseline arousal levels alone. To assess this, and to identify the timescale (short- or long-) at which changes in arousal best predicted attentional measures, we compared models with epochs of varying durations for capturing arousal and attention activity. Short epochs highlight rapidly changing phasic activity in the signal, whereas longer timescales of activity average over more rapid fluctuations to highlight slower moving trends or "states" in the signal. Within each timescale, we compared the predictive value between epoch-specific arousal levels and whole-session average arousal. Whole session average arousal corresponds to a single epoch of approximately 20 minutes, which is the longest approximation of arousal for the session. For this reason we refer to it as the tonic arousal level.

Specifically, we tested a range of timescales from 2-seconds to 360 seconds (six minutes) to assess which of these provided the most predictive power for capturing associations between arousal and look duration. We chose seven timescales in total, binning the whole-session data into epochs of 2, 5, 10, 20, 60, 120 and 360 seconds apiece. For each timescale, we created a pair of models. The 
first model tested the contribution of only the whole-session tonic arousal data for predicting the looking duration values at each epoch length. This model indicates how well, across the entire session, tonic arousal levels can predict the look duration data averaged within each epoch. The second model includes the whole-session tonic arousal as a predictor as well as the average arousal value calculated within each individual epoch (phasic). By comparing the relative variance explained between each pair of models, we see to what extent arousal fluctuations at that timescale help predict anything over and above the tonic baseline arousal measure. Next, by comparing the additional variance predicted by the phasic (epoch-specific) components across the models of different timescales, we can identify the epoch at which arousal makes the strongest predictions for the attentional variable.

Prior to conducting calculations, all data were checked to ensure that assumptions of homoscedasticity and non-collinearity were met. Table 1 shows the model parameters, and the $\mathrm{R}^{2}$, for the pair of models that were fitted at each different epoch length. Looking at the two models created for each epoch, we see that tonic arousal levels significantly predicted look duration (model 1), and that when this tonic arousal was controlled for, per-epoch arousal also significantly predicted looking behavior $(\mathrm{p}<.05)$. The negative regression coefficients $\beta$ values) show that periods of higher arousal are associated with shorter looking times, consistent with the results from analyses 1 and 2. Figure 3 shows the increase in $\mathrm{R}^{2}$ observed between the two models. Comparing the two models created for each epoch, per-epoch arousal added between 0 and 9 percent additional explained variance. At long epoch durations there was no observed increase in $\mathrm{R}^{2}$ between the two models. This is unsurprising, since at long epoch durations, the average arousal within that epoch begins to more closely approximate the average arousal across the entire session. At shorter epoch 
Running head: DYNAMICS OF AROUSAL AND ATTENTION

durations, however, the $\Delta \mathrm{R}^{2}$ values increase. This suggests that short-term changes in arousal are explaining more variance in look duration than are baseline arousal levels. This difference peaks at 20 seconds, suggesting that the predictive value of phasic arousal with phasic look duration is greatest on a 20 -second scale. At epoch durations shorter than 20 seconds, $\Delta \mathrm{R}^{2}$ values decrease.

INSERT TABLE 1 HERE.

INSERT FIGURE 3 HERE. 


\section{Discussion}

We examined dynamic changes in arousal and their effects on moment to moment changes in attentional measures across a 20-minute testing session administered to 12 month old infants. Based on theories derived from animal studies (Aston-Jones et al., 1999; see also de Barbaro et al., 2011), we predicted that increases in arousal would correspond to a more vigilant attentional profile, indexed by short look durations.

Our first analysis considers variations in arousal and look duration within individual infants. By splitting each infant's session into one-second epochs we can observe how dynamic fluctuations in arousal and attention are related. Considering every such epoch as a datapoint, the average correlation between arousal and attention measured simultaneously i.e. timelag $(t=0)$ was $r=-.22$ for epoched looks (Figure 1c). That is, periods of high arousal within a session are associated with shorter looks, whereas periods of low arousal within a session are associated with longer looks. Of note, this analysis controls, via z-scoring, for baseline differences in both the attention and arousal measures. Thus, these results cannot be accounted for by stable differences in the baselines activity between infants.

We also found that arousal levels up to 25 seconds before the onset of a look are predictive of the duration of a look, whereas the reverse is not true (Figure 1c). What can we make of this asymmetry? One possibility is that both attention and arousal are both downstream effects of some common central signaling e.g. the Locus Coeruleus), perhaps responding to changes in novelty and uncertainty. The fact that arousal predicts attention but not vice versa could reflect the fact 
that peripheral arousal levels change more rapidly than our attentional measures. However, the autocorrelations indicate that our arousal composite changes more slowly than look durations (Figure 1a, 1b), so this seems unlikely.

We also examined the possibility that both our arousal and attentional measures may be sensitive to changes in the media that the infant is engaging with. We know that infants show longer looks while observing complex or dynamic images as well as films, and that they show shorter looks when observing static or simpler images (Courage et al., 2006; Wass \& Smith, 2014). It is possible that, during a session, looking measures are independently affected by differences between the tasks as well as by changing arousal levels. To control for any differences in task features across the session, in Analysis 2 we therefore considered attentional changes within a single task, in which each look corresponded to an on-screen gaze to a static image of a child's face.

We elected to use a slightly different analysis for this task, considering the relation between the duration of each look, and the average arousal for the duration of that look for each individual infant, across three blocks of familiarization. By considering the data look-by-look rather than epoch-by-epoch we highlight that the arousal within the look itself can predict variation across looks within individual infants' data, in addition to the preceding or following seconds. Figure 2 indicates that the majority of infants showed a significant negative relation between looks and arousal.

In Analysis 3 we fitted a series of linear mixed-effects models to our data to test the relative import of different timescales of arousal activity for predicting attention. In particular, we evaluated 
whether phasic arousal levels are more predictive of look duration than tonic baseline) arousal levels. The amount of variation in the look duration data that was accounted for by phasic changes in arousal (above and beyond that accounted for by baseline arousal levels) was found to be greatest when a 20-second epoch duration was used. At longer epochs, more fluctuations in attention are averaged into each epoch so that rapid arousal fluctuations become overshadowed. The results of this analysis are consistent with those of Analysis 2, suggesting significant covariation of changes in arousal and attention on a similar time-scale.

Why was a 20 -second epoch duration found to be most predictive? This may be because these analyses are zero-lagged (i.e. they compare arousal at one moment in time with look duration at the same moment in time). As we showed in Analysis 1, changes in arousal tend to precede shortterm changes in look duration by several seconds. This time-lag effect may have reduced the relations found at shorter epoch durations in Analysis 3.

While arousal figures prominently in many theories of attention (e.g. Richards, 2010) there has been a lack of data considering the dynamic processes of arousal and its moment-to-moment effects on cognition. Instead, the majority of data collected has averaged over these dynamic processes in order to obtain "more pure" or "cleaner" cognitive measures. Theories and models within cognitive science have long indicated that the situational and embodied nature of cognitive activity merits consideration and is indeed part and parcel of the cognitive process (Clark, 2008; Hutchins, 1995; see also de Barbaro, Johnson, Forster, \& Deak, 2013). Moreover, neurobiological accounts of what are traditionally considered pure/cool "cognitive" tasks - such as executive functioning and attentional control - are increasingly indicating the role of these embodied 
practices, and specifically the manner in which affect qualitatively changes the nature of neural activity in these classic cognitive domains.

The lack of dynamic data on attention and arousal contributes to the paucity of theories considering the dynamics of these processes. Stability in looking measures is taken as evidence of static differences in faster vs. slower speed of information encoding. However, dynamic accounts could also account for these findings. An alternative theory from a more enactive cognition perspective (Makeig, Gramann, Jung, Sejnowski, \& Poizner, 2009) posits that activity is a function the fit between an individual's current state and the affordances of their environment. The Aston-Jones model specifically highlights that an individuals' threshold for responding to the surround (i.e. how quickly they may respond to a stimulus, how long they engage with a stimulus, and the balance between dampening down peripheral stimuli and focusing on a single central focus) has to do with dynamically changing assessments of the novelty or uncertainty of the environment, as integrated and tracked by Locus Coeruleus activity or sympathetic nervous system activity more broadly.

This means that the amount of time that an infant looks at a stimulus can reflect something about their dynamically changing internal state. Thus, while this framework does not affect the validity of past findings using looking time as a main dependent measure, it may affect their interpretation. At low arousal levels corresponding to calm states, infants may have a higher threshold for peripheral stimulation and may thus engage longer with central stimuli, whereas at higher arousal levels they may show faster orienting patterns and have a higher readiness to engage with many stimuli quickly, manifesting in shorter looks. In neither case would we say that infants have 
encoded the stimulus "fully", as there is no discrete total amount of information to be processed in a stimulus, in the same way that there are different styles of appreciating art at a gallery (Aslin, 2007). Previous developmental findings have also indicated that short looks should not simply be interpreted as fast or "complete" processing of stimuli (e.g. see Kagan, 2002 for a review; Kidd, Piantadosi, \& Aslin, 2012). Finally, infants exhibiting more reactivity or more vigilant attentional behaviors may be able to recognize the most salient differences between habituated and novel stimuli but this does not mean that their engagement or what they have learned is the same as that of longer-looking infants (see, e.g. Rose, Feldman, \& Jankowski, 2001; Schöner \& Thelen, 2006).

Distributed and situated accounts of cognition indicate that the details of embodied sensorimotor experience, including the activity of the body's arousal and regulation systems, can give us novel clues about the learning process. These accounts also highlight the role of the micro-dynamics of cognitive activity (de Barbaro, Johnson, Forster, \& Deák, in press; Goldstein \& Schwade, 2008; Yu \& Smith, 2012). As we gain novel technologies and computational means for capturing and analyzing these dynamics, we are able to characterize more about these rich processes (de Barbaro et al., 2013; Yu, Yurovsky, \& Xu, 2012). Importantly, where static measures indicate crystallization of the developmental system, dynamic measures invite us to consider processes in flux or that have not yet stabilized - arguably the goal of a developmental science. 
Running head: DYNAMICS OF AROUSAL AND ATTENTION

\section{Tables}

Table 1. Model parameters for the two linear mixed-effects models that were fitted. Details of the two models are given in the main text.

\begin{tabular}{|c|c|c|c|c|c|c|c|c|c|}
\hline & \multirow{2}{*}{\multicolumn{3}{|c|}{$\begin{array}{l}\text { Model } 1 \text { - Tonic } \\
\text { Tonic component }\end{array}$}} & \multicolumn{6}{|c|}{ Model 2 - Tonic + Phasic } \\
\hline & & & & & compc & ent & & $\begin{array}{l}\text { sic com } \\
\mathrm{R}^{2}\end{array}$ & ent \\
\hline $\begin{array}{l}\text { Epoch } \\
\text { Dur. } \\
\text { secs) }\end{array}$ & $\beta$ & $t$ & $p$ & $\beta$ & $t$ & $p$ & $\beta$ & $t$ & $p$ \\
\hline 2 & -0.5 & -3.1 & 0.003 & -0.05 & -0.31 & 0.76 & -0.51 & -38.19 & 0.001 \\
\hline 5 & -1.02 & -4.1 & 0.001 & -0.35 & -1.4 & 0.17 & -0.68 & -35.5 & 0.001 \\
\hline 10 & -1.02 & -4.2 & 0.001 & -0.32 & -1.3 & 2.1 & -0.72 & -28.1 & 0.001 \\
\hline 20 & -1.05 & -4.4 & 0.001 & -0.32 & -1.3 & 0.2 & -0.74 & -21.6 & 0.001 \\
\hline 60 & -1.05 & -4.3 & 0.001 & -0.42 & -0.17 & 0.098 & -0.63 & -11.4 & 0.001 \\
\hline 120 & -1.07 & -4.2 & 0.001 & -0.45 & -1.7 & 0.09 & -0.61 & -8.3 & 0.001 \\
\hline 360 & -0.11 & -3.3 & 0.001 & -0.67 & -2.2 & 0.03 & -0.33 & -2.5 & 0.01 \\
\hline
\end{tabular}




\section{Figures}
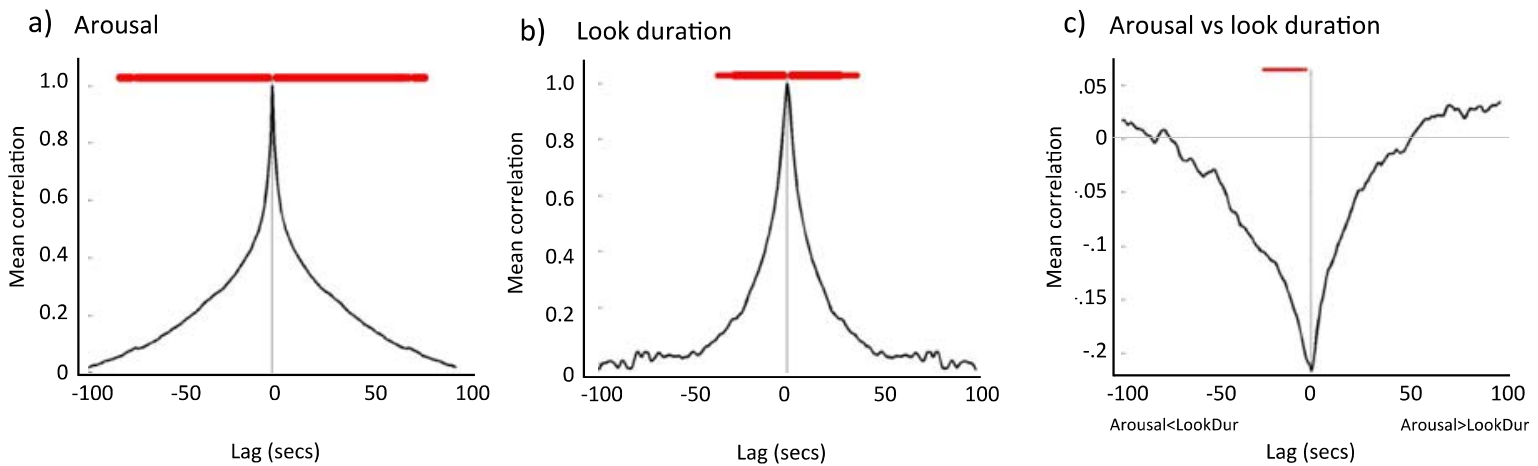

Figure 1: a) and b) Results of auto-correlations for a) arousal and b) look duration. X-axis indicates the time-lag. Y-axis indicates the correlation observed between that variable and itself at that time-lag. c) Results of cross-correlations between arousal and look duration. X-axis indicates the time-lag. Y-axis indicates the correlation observed between the two variables, when one was time-lagged relative to the other. Coloured dots above the plot indicate whether a significant relation was observed at each time-lag $p<.05)$ 


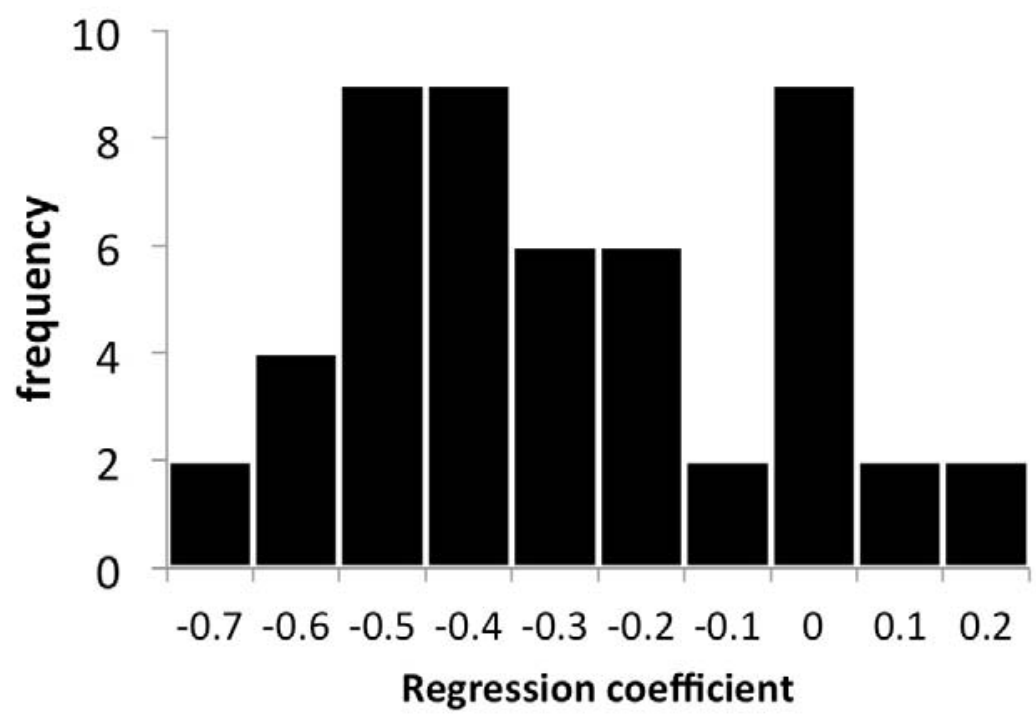

Figure 2: Participant by participant, we examined the correlation between individual looks recorded during a habituation experiment and the participants' arousal during that look. Regression coefficients observed are plotted as a histogram. In almost all participants, negative correlations were observed. 


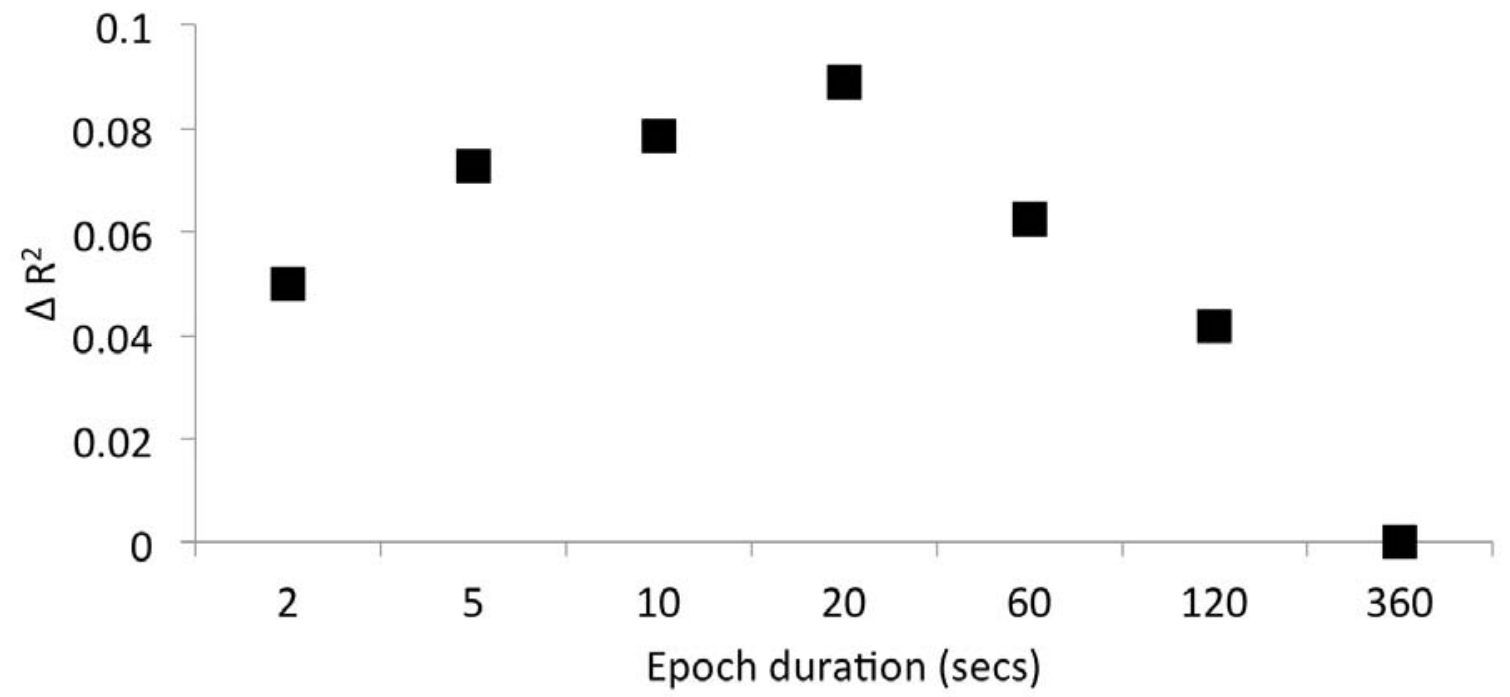

Figure 3: $Y$-axis shows $\Delta R^{2}$ - i.e. the degree to which adding the per-epoch variable explains additional variance to that explained by tonic arousal. The procedure for calculating this is described in the main text. 
Running head: DYNAMICS OF AROUSAL AND ATTENTION

\section{References}

Arnsten, A. F. (2009). Stress signalling pathways that impair prefrontal cortex structure and function. Nature Reviews Neuroscience, 10(6), 410-422.

Arnsten, A. F. T. (1998). NEUROSCIENCE: Enhanced: The Biology of Being Frazzled. Science, 280(5370), 1711.

Arnsten, A. F. T., \& Li, B. M. (2005). Neurobiology of Executive Functions: Catecholamine Influences on Prefrontal Cortical Functions. Biological Psychiatry, 57(11), 1377-1384.

Aslin, R. N. (2007). What's in a look? Developmental Science, 10(1), 48-53. doi: 10.1111/j.14677687.2007.00563.x

Aston-Jones, G., \& Bloom, F. E. (1981). Nonrepinephrine-containing locus coeruleus neurons in behaving rats exhibit pronounced responses to non-noxious environmental stimuli. J. Neurosci., 1(8), 887900.

Aston-Jones, G., Chiang, C., \& Alexinsky, T. (1991). Discharge of noradrenergic locus coeruleus neurons in behaving rats and monkeys suggests a role in vigilance. Progress in brain research, 88, 501520.

Aston-Jones, G., \& Cohen, J. D. (2005). An integrative theory of locus coeruleus-norepinephrine function: adaptive gain and optimal performance. Annual Review of Neuroscience, 28(1), 403-450.

Aston-Jones, G., Rajkowski, J., \& Cohen, J. (1999). Role of locus coeruleus in attention and behavioral flexibility. Biological Psychiatry, 46(9), 1309-1320.

Blair, C., Granger, D., Willoughby, M., Mills-Koonce, R., Cox, M., Greenberg, M., Kivlighan, K., \& Fortunato, C. (2011). Salivary cortisol mediates effects of poverty and parenting on executive functions in early childhood. Child Development, 82(6), 1970-1984.

Bronson, G. (1974). The postnatal growth of visual capacity. Child Development, 873-890.

Carlson, E. A., Jacobvitz, D., \& Sroufe, L. A. (1995). A developmental investigation of inattentiveness and hyperactivity. Child Development, 66(1), 37-54.

Clark, A. (2008). Supersizing the mind: Embodiment, action, and cognitive extension: Oxford University Press, USA.

Clifford, P., Richardson, S., \& Hemon, D. (1989). Assessing the Significant of the Correlation Between Two Spatial Processes. Biometrics, 45(1), 123-134. doi: 10.2307/2532039

Colombo, J., \& Cheatham, C. L. (2006). The emergence and basis of endogenous attention in infancy and early childhood. Advances in child development and behavior, 34, 283-322.

Colombo, J., \& Mitchell, D. W. (1990). Individual differences in early visual attention: fixation time and information processing. In J. Colombo \& J. W. Fagan (Eds.), Individual Differences in Infancy. Hillsdale, N.J.: Erlbaum.

Colombo, J., \& Mitchell, D. W. (2009). Infant visual habituation. Neurobiology of learning and memory, 92(2), 225-234. doi: 10.1016/j.nlm.2008.06.002

Courage, M., Reynolds, G., \& Richards, J. (2006). Infants' attention to patterned stimuli: Developmental change from 3 to 12 months of age. Child Development, 77(3), 680-695.

de Barbaro, K., Chiba, A., \& Deák, G. O. (2011). Micro-analysis of infant looking in a naturalistic social setting: insights from biologically based models of attention. Developmental Science, 14(5), 1150-1160.

de Barbaro, K., Johnson, C. M., Forster, D., \& Deák, G. (in press). Infant Sensory-Motor Decoupling Contributes To 12 Month Social "Revolution": A Longitudinal Investigation Of Mother-InfantObject Interactions. Child Development. 
de Barbaro, K., Johnson, C. M., Forster, D., \& Deak, G. O. (2013). Methodological Considerations For Investigating the Micro-Dynamics of Social Interaction Development. IEEE Transactions on Autonomous Mental Development, 5(3), 258-270.

Field, T. M. (1981). Infant gaze aversion and heart rate during face-to-face interactions*. Infant Behavior and Development, 4, 307-315.

Freibergs, V., \& Douglas, V. I. (1969). Concept learning in hyperactive and normal children. Journal of Abnormal Psychology, 74(3), 388.

Gardner, J. M., \& Karmel, B. Z. (1995). Development of arousal-modulated visual preferences in early infancy. Developmental Psychology, 31(3), 473.

Geva, R., Gardner, J. M., \& Karmel, B. Z. (1999). Feeding-based arousal effects on visual recognition memory in early infancy. Developmental Psychology, 35(3), 640.

Goldstein, M. H., \& Schwade, J. A. (2008). Social feedback to infants' babbling facilitates rapid phonological learning. Psychological Science, 19(5), 515.

Hastings, J. E., \& Barkley, R. A. (1978). A review of psychophysiological research with hyperkinetic children. Journal of abnormal child psychology, 6(4), 413-447.

Hutchins, E. (1995). Cognition in the Wild: MIT press Cambridge, MA.

Johnson, M. H. (1990). Cortical maturation and the development of visual attention in early infancy. Journal of Cognitive Neuroscience, 2(2), 81-95.

Kagan, J. (2002). Surprise, uncertainty, and mental structures. . Cambridge, MA: Harvard University Press.

Karmel, B. Z., Gardner, J. M., \& Magnano, C. L. (1991). Attention and arousal in early infancy.

Kidd, C., Piantadosi, S. T., \& Aslin, R. N. (2012). The Goldilocks effect: Human infants allocate attention to visual sequences that are neither too simple nor too complex. PloS one, 7(5), e36399.

Makeig, S., Gramann, K., Jung, T.-P., Sejnowski, T. J., \& Poizner, H. (2009). Linking brain, mind and behavior. International Journal of Psychophysiology, 73(2), 95-100.

Rajkowski, J., Kubiak, P., \& Aston-Jones, G. (1994). Locus coeruleus activity in monkey: Phasic and tonic changes are associated with altered vigilance. Brain Research Bulletin, 35(5-6), 607-616.

Richards, J. E. (2010). The development of attention to simple and complex visual stimuli in infants: Behavioral and psychophysiological measures. Developmental Review, 30(2), 203-219. doi: 10.1016/j.dr.2010.03.005

Richards, J. E., \& Casey, B. J. (1991). Heart rate variability during attention phases in young infants. Psychophysiology, 28(1), 43-53.

Rose, S. A., Feldman, J. F., \& Jankowski, J. J. (2001). Attention and recognition memory in the 1st year of life: a longitudinal study of preterm and full-term infants. Developmental Psychology, 37(1), 135.

Rose, S. A., Feldman, J. F., \& Jankowski, J. J. (2002). Processing speed in the 1st year of life: A longitudinal study of preterm and full-term infants. Developmental Psychology, 38(6), 895-902. doi: 10.1037//0012-1649.38.6.895

Schöner, G., \& Thelen, E. (2006). Using dynamic field theory to rethink infant habituation. Psychological Review, 113(2), 273.

Sokolov, E. N. (1963). Perception and the conditioned reflex. New York: MacMillan.

Tang, Y. Y., Rothbart, M. K., \& Posner, M. I. (2012). Neural correlates of establishing, maintaining, and switching brain states. Trends in Cognitive Sciences, 16(6), 330-337.

Wass, S. V., \& Smith, T. J. (2014). Individual Differences in Infant Oculomotor Behavior During the Viewing of Complex Naturalistic Scenes. . Infancy, 19(4), 352-384.

Waters, E., Matas, L., \& Sroufe, L. A. (1975). Infants' reactions to an approaching stranger: Description, validation, and functional significance of wariness. Child Development, 348-356.

Yu, A. J., \& Dayan, P. (2003). Expected and unexpected uncertainty: ACh and NE in the neocortex. Advances in Neural Information Processing Systems, 15, 157-164. 
Running head: DYNAMICS OF AROUSAL AND ATTENTION

Yu, C., \& Smith, L. B. (2012). Embodied attention and word learning by toddlers. Cognition.

Yu, C., Yurovsky, D., \& Xu, T. L. (2012). Visual data mining: An exploratory approach to analyzing temporal patterns of eye movements. Infancy, 17(1), 33-60.

Zentall, S. (1975). Optimal stimulation as theoretical basis of hyperactivity. American Journal of Orthopsychiatry, 45(4), 549. 